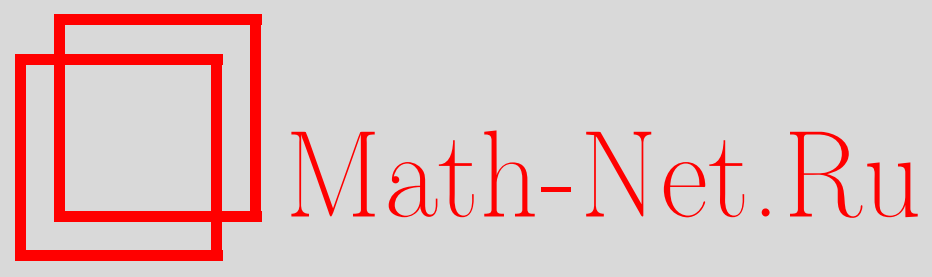

Х. Д. Икрамов, А. А. Чесноков, О матрицах, обратных к брауновским и родственным им матрицам, Матем. заметкu, 2004, том 75, выпуск 1, 89-99

DOI: https://doi.org/10.4213/mzm9

Использование Общероссийского математического портала Math-Net.Ru подразумевает, что вы прочитали и согласны с пользовательским соглашением http://www.mathnet.ru/rus/agreement

Параметры загрузки:

IP : 3.82 .47 .9

26 апреля 2023 г., 04:50:09 


\title{
О МАТРИЦАХ, ОБРАТНЫХ К БРАУНОВСКИМ \\ И РОДСТВЕННЫМ ИМ МАТРИЦАМ
}

\author{
Х. Д. Икрамов, А. А. Чесноков
}

\begin{abstract}
В нескольких статьях $\Phi$. Валви указал достаточные условия для того, чтобы брауновские и родственные им матрицы имели хессенберговы обратные. Мы интерпретируем эти условия с точки зрения известных фактов о матрицах малого треугольного ранга, что позволяет сформулировать более общие утверждения о хессенберговости обратной матрицы. Кроме того, при некотором естественном условии “неразложимости" найден явный вид матрицы, обратной к брауновской. Он схож с хорошо известным явным видом матрицы, обратной к неразложимой трехдиагональной матрице. При этом параметры, определяющие обратную к $(n \times n)$-матрице, могут быть вычислены за $O(n)$ арифметических операций.

Библиографоия: 14 названий.
\end{abstract}

1. Введение. Брауновской называется $(n \times n)$-матрища следующего строения:

$$
A=\left(\begin{array}{ccccc}
a_{1} & b_{1} & b_{1} & \ldots & b_{1} \\
c_{1} & a_{2} & b_{2} & \ldots & b_{2} \\
c_{1} & c_{2} & a_{3} & \ldots & b_{3} \\
\vdots & \vdots & \vdots & \ddots & \vdots \\
c_{1} & c_{2} & c_{3} & \ldots & a_{n}
\end{array}\right)
$$

Таким образом, $A$ определяется всего лишь $3 n-2$ параметрами. Матрицу, получаемую из матришы (1) отражением относительно ее центра, будем называть отраженной брауновской. Обе разновидности брауновских матриц обладают одинаковыми свойствами (возможно, с точностью до указанной центральной симметрии).

Брауновские матрицы издавна используются как тестовые. Например, в известной книге [1] матрища 3.24 является брауновской, а матрицы $3.12,3.13$ и 4.14 - отраженньми брауновскими.

В статье 1977 г. [2] Валви показал, что симметричная (отраженная) брауновская матрица вида

$$
K=\left(\begin{array}{ccccc}
k_{1} & k_{2} & k_{3} & \ldots & k_{n} \\
k_{2} & k_{2} & k_{3} & \ldots & k_{n} \\
k_{3} & k_{3} & k_{3} & \ldots & k_{n} \\
\vdots & \vdots & \vdots & \ddots & \vdots \\
k_{n} & k_{n} & k_{n} & \ldots & k_{n}
\end{array}\right)
$$


если она невырождена, имеет трехдиагональную обратную. (В дальнейшем невырожденность интересующих нас матриц предполагается без специального упоминания.) Позднее в [3] это наблюдение было обобщено: оказалось, что для матрищы типа

$$
A=\left(\begin{array}{ccccc}
a_{1} & b_{1} & b_{1} & \ldots & b_{1} \\
a_{1} & a_{2} & b_{2} & \ldots & b_{2} \\
a_{1} & a_{2} & a_{3} & \ldots & b_{3} \\
\vdots & \vdots & \vdots & \ddots & \vdots \\
a_{1} & a_{2} & a_{3} & \ldots & a_{n}
\end{array}\right)
$$

обратная матрища $B=A^{-1}$ является верхней хессенберговой, т.е. $b_{i j}=0$ при $i-j>1$.

Еще один любопытньй результат, содержащийся в [2], состоит в том, что трехдиагональную форму имеет обратная для симметричной матрицы вида

$$
\left(\begin{array}{ccccc}
k_{1} a_{1} & k_{2} a_{1} & k_{3} a_{1} & \ldots & k_{n} a_{1} \\
k_{2} a_{1} & k_{2} a_{2} & k_{3} a_{2} & \ldots & k_{n} a_{2} \\
k_{3} a_{1} & k_{3} a_{2} & k_{3} a_{3} & \ldots & k_{n} a_{3} \\
\vdots & \vdots & \vdots & \ddots & \vdots \\
k_{n} a_{1} & k_{n} a_{2} & k_{n} a_{3} & \ldots & k_{n} a_{n}
\end{array}\right) .
$$

Последняя представляет собой адамарово (т.е. поэлементное) произведение отраженной брауновской матрицы (2) и брауновской матрицы типа (3). В [4] и этот результат был обобщен: для адамарова произведения матрицы (2) и несимметричной матрицы (3) обратная матрица имеет верхнюю форму Хессенберга.

Цель настоящей статьи заключается в том, чтобы объяснить указанные результаты в свете общих фактов о матрицах малого вне- и поддиагонального рангов. Авторы работ [2]-[4] не были знакомы (а в некоторых случаях, исходя из хронологии, и не могли быть знакомы) с этими фактами. Кроме того, при некотором естественном ограничении на брауновскую матрицу (1) мы указьваем явньй вид обратной к ней матрицы, аналогичный хорошо известному виду матрицы, обратной к неразложимой трехдиагональной матрице. При этом параметры, определяющие обратную матрищу, могут быть вычислены за $O(n)$ операций.

Хотя брауновские матрицы составляют довольно узкий и экзотический матричньй класс, приемы, используемые при работе с ними, получили далекое развитие в таких важных вопросах современной линейной алгебры, как семисепарабельность [5], мозаичные ранги [6], и др.

2. Структурные ранги взаимно обратных матриц. Пусть $A$ - квадратная матрица порядка $n$. Внедиагональным рангом $w(A)$ этой матрицы называется наивысший порядок ее ненулевых миноров, целиком составленных из внедиагональных элементов. Для диагональной матрицы $D$ (единственная ситуация, когда все указанные миноры равны нулю) полагаем $w(D)=0$.

Для невырожденной диагональной матрицы $D$ обратная матрица $D^{-1}$, очевидно, тоже диагональна, поэтому

$$
w\left(D^{-1}\right)=w(D)=0 .
$$

Как установлено в [7], равенство внедиагональных рангов взаимно обратных матрищ является общим правилом. 
Теорема 1. Пусть А-невырожденная квадратная матрица. Тогда

$$
w\left(A^{-1}\right)=w(A) .
$$

Матрица, обратная к треугольной матрице $A$, сама является треугольной, причем верхней (или нижней), если $A$ - верхняя (нижняя) треугольная. Этот элементарный факт получил в [7] нетривиальное обобщение. Назовем верхнетреугольным (нижнетреугольным) рангом квадратной матрицы $A$ наивысший порядок ее ненулевых миноров, целиком составленных из наддиагональных (подлиагональных) элементов. Верхнетреугольньй (нижнетреугольньй) ранг нижнетреугольной (верхнетреугольной) матрицы $A$ по определению равен нулю. В общем случае, верхнетреугольньй ранг $A$ обозначаем символом $r_{u}(A)$, а нижнетреугольный ранг - символом $r_{l}(A)$.

Теорема 2 ([8]). Пусть $A$ - невырожденная квадратная матрица. Тогда

$$
\begin{aligned}
r_{u}\left(A^{-1}\right) & =r_{u}(A), \\
r_{l}\left(A^{-1}\right) & =r_{l}(A) .
\end{aligned}
$$

Нетрудно видеть, что оба треугольных ранга каждой из матрищ (1)-(4) не превосходят 1 и равны единице, если эти матрицы не являются треугольными.

СлЕдСТВИЕ 1. Если невырожденная брауновская матрица $A$ не является треугольной, то

$$
r_{u}\left(A^{-1}\right)=r_{l}\left(A^{-1}\right)=1
$$

СлеДСТВИЕ 2. Пусть $A_{1}$ - брауновская, а $A_{2}$ - отражсенная брауновская матрицы, причем обе невырожденны и не являются треугольными. Тогда для адамарова произведения $A=A_{1} \circ A_{2}$ имеем

$$
r_{u}\left(A^{-1}\right)=r_{l}\left(A^{-1}\right)=1 .
$$

Классическая ситуация, в которой реализуется треугольный ранг, равный единице, связана с хессенберговыми матрицами.

СледСТВИЕ 3. Пусть $A$ - невырожденная матрица Хессенберга, не являющаяся треугольной. Если $A$ - верхняя хессенбергова матрииа, то

$$
r_{l}\left(A^{-1}\right)=1
$$

Для нижсней хессенберговой матричь А имеем

$$
r_{u}\left(A^{-1}\right)=1
$$

Если $A$ - трехдиагональная матрица, то выполняются оба равенства (10) и (11).

Посмотрим, как вьглядит матрища, обратная к хессенберговой матрице $A$. Это даст нам представление об устройстве матрищы, обратной к брауновской.

Для определенности возьмем нижнюю матрицу Хессенберга порядка $n$, причем будем считать ее неразложимой, т.е.

$$
a_{i j} \neq 0 \quad \text { при } j=i+1 .
$$


Представим $A$ в блочной форме

$$
A=\left(\begin{array}{cc}
b & A_{n-1} \\
\alpha & c^{T}
\end{array}\right)
$$

где $A_{n-1}-$ квадратная матрица порядка $n-1$. В силу предположения (12) эта треугольная подматрица невырожденна. Используя форму Банахиевича-Шура для обратной матрицы (один из вариантов этой формы можно найти в [9, раздел 0.7.3]) имеем

$$
A^{-1}=\left(\begin{array}{cc}
-\frac{1}{s} c^{T} A_{n-1}^{-1} & \frac{1}{s} \\
A_{n-1}^{-1}+\frac{1}{s} A_{n-1}^{-1} b c^{T} A_{n-1}^{-1} & -\frac{1}{s} A_{n-1}^{-1} b
\end{array}\right)
$$

где число

$$
s=\alpha-c^{T} A_{n-1}^{-1} b
$$

есть дополнение Шура подматрицы $A_{n-1}$. Вводя $n$-мерные векторы

$$
u=\frac{1}{s}\left(\begin{array}{c}
1 \\
-A_{n-1}^{-1} b
\end{array}\right)
$$

и

$$
v^{T}=\left(\begin{array}{ll}
-c^{T} A_{n-1}^{-1} & 1
\end{array}\right)
$$

можем переписать (14) в виде

$$
A^{-1}=\left(\begin{array}{cc}
0 & 0 \\
A_{n-1}^{-1} & 0
\end{array}\right)+u v^{T}
$$

Для хессенберговой матрицы $A$ подматрица $A_{n-1}$ нижнетреугольная; следовательно, нижнетреугольной является и $A_{n-1}^{-1}$. Поэтому диагональная и наддиагональная части матрищы (18) совпадают с соответствующими частями одноранговой матрицы $u v^{T}$, откуда

$$
\left(A^{-1}\right)_{i j}=u_{i} v_{j}, \quad j \geqslant i
$$

Отметим, что эти рассуждения заимствованы нами из статьи [10].

Для верхней матрищы Хессенберга аналогичным образом определяются диагональные и поддиагональные элементы в $A^{-1}$. Если же $A$-неразложимая трехдиагональная матрица, т.е.

$$
a_{i j} \neq 0 \quad \text { при }|j-i|=1,
$$

то найдутся $n$-мерные векторы $x, y, u, v$ такие, что

$$
\left(A^{-1}\right)_{i j}= \begin{cases}u_{i} v_{j}, & j \geqslant i \\ y_{i} x_{j}, & i \geqslant j .\end{cases}
$$

Тем самьм должны вьполняться условия согласования

$$
u_{i} v_{i}=x_{i} y_{i}, \quad i=1,2, \ldots, n
$$


Заметим, что матрицу (21) можно интерпретировать как адамарово произведение брауновской матрицы

$$
\left(\begin{array}{cccc}
u_{1} & u_{1} & \ldots & u_{1} \\
x_{1} & u_{2} & \ldots & u_{2} \\
\vdots & \vdots & \ddots & \vdots \\
x_{1} & x_{2} & \ldots & u_{n}
\end{array}\right)
$$

относящейся (с точностью до транспонирования) к типу (3), и отраженной брауновской матрицы

$$
\left(\begin{array}{cccc}
v_{1} & v_{2} & \ldots & v_{n} \\
y_{2} & v_{2} & \ldots & v_{n} \\
\vdots & \vdots & \ddots & \vdots \\
y_{n} & y_{n} & \ldots & v_{n}
\end{array}\right)
$$

того же типа. Это наблюдение было сделано в [11].

Исходя из следствия 1 , можно ожидать, что матрица, обратная к брауновской, имеет сходную форму, по крайней мере, при ограничении, аналогичном условию неразложимости в трехдиагональном случае. Мы вернемся к этому вопросу в разделе 4.

3. Хессенберговость обратной матрицы. Свойство обратной матрицы быть ленточной можно установить, выполняя некоторые проверки для рангов подматриц в самой матрище $A$. Мы воспроизведем соответствующий результат из [12], адаптируя его для случая хессенберговой формы.

Теорема 3. Пусть $A$ - невырожсденная $(n \times n)$-матрица. Тогда следующие два утверждения әквивалентны:

1) $A^{-1}$ имеет верхнюю форму Хессенберга;

2) любая подматрича матрииы $A$, расположсенная в области $i \geqslant j$, имеет ранг, меньший или равный единице.

Проверки, предусматриваемые утверждением 2 , можно упростить, пользуясь дополнением теоремы 3 , найденным в [10]. Обозначим через $A(r, s)$ подматрицу в $A$, образованную ее последними $r$ строками и первыми $s$ столбцами.

ТЕорема 4. В условиях теоремы 3 каждое из утверждений 1) и 2) эквивалентно соотношениям

$$
\operatorname{rank} A(r, n+1-r)=1, \quad 2 \leqslant r \leqslant n-1
$$

Если для матрицы $A$ выполнены равенства (22) и при этом

$$
a_{n 1} \neq 0
$$

то $A^{-1}$ является неразложимой верхней хессенберговой матрицей.

Применим теорему 4 к выяснению условий, при которых брауновская матрища (1) имеет (верхнюю) хессенбергову матрицу $A^{-1}$. При этом будем считать, что $c_{1} \neq 0$, так как в противном случае хессенберговость $A^{-1}$ определяется свойствами нижней угловой подматрицы порядка $n-1$ в $A$. 
Равенство (22) при $r=2$ требует, чтобы подматрица

$$
\left(\begin{array}{ccccc}
c_{1} & c_{2} & \ldots & c_{n-2} & a_{n-1} \\
c_{1} & c_{2} & \ldots & c_{n-2} & c_{n-1}
\end{array}\right)
$$

имела ранг 1. Очевидно, это требование равносильно тому, чтобы

$$
c_{n-1}=a_{n-1}
$$

Аналогичным образом остальные соотношения (22) приводят к равенствам

$$
c_{i}=a_{i}, \quad i=2, \ldots, n-2
$$

Мы приходим к следующему утверждению.

ТЕорема 5. Для того чтобы невырожденная брауновская матрица (1) имела верхнюю хессенбергову матричу $A^{-1}$, необходимо и достаточно, чтобы выполнялись равенства

$$
c_{i}=a_{i}, \quad i=2, \ldots, n-1 .
$$

При $c_{1} \neq 0$ хессенбергова матрица $A^{-1}$ является неразложсимой.

Теорема 5 полностью объясняет результаты из [2], [3], относящиеся к брауновским матрицам. При этом она показьвает избыточность выдвинутых там условий. Действительно, можно заменить диагональные элементы $k_{n}$ в $(2)$ и $a_{1}$ в $(3)$ произвольньми числами, лишь бы матрища оставалась невырожденной, и это не повлияет на трехдиагональность или хессенберговость формы обратной матрицы.

Пусть, например,

$$
A=\left(\begin{array}{lllll}
7 & 3 & 3 & 3 & 3 \\
1 & 4 & 7 & 7 & 7 \\
1 & 4 & 6 & 8 & 8 \\
1 & 4 & 6 & 5 & 7 \\
1 & 4 & 6 & 5 & 1
\end{array}\right)
$$

т.е. $a_{1}=7 \neq c_{1}=1$. Имеем

$$
A^{-1}=\left(\begin{array}{ccccc}
\frac{4}{25} & \frac{6}{25} & -\frac{3}{25} & -\frac{4}{25} & -\frac{2}{25} \\
-\frac{1}{25} & -\frac{39}{25} & \frac{46}{75} & \frac{184}{225} & \frac{92}{225} \\
0 & 1 & -\frac{2}{3} & -\frac{2}{9} & -\frac{1}{9} \\
0 & 0 & \frac{1}{3} & -\frac{7}{18} & \frac{1}{18} \\
0 & 0 & 0 & \frac{1}{6} & -\frac{1}{6}
\end{array}\right)
$$

Таким образом, $A^{-1}$ - хессенбергова матрица.

Обратимся теперь к адамаровым произведениям брауновских матриц. Прежде всего, отметим простое, но полезное следствие теоремы 5. 
СлЕДСТВИЕ 4. Пусть $A_{1} u A_{2}$ - невырожденные брауновские матрицы, имеющие верхние хессенберговы обратные матрицы $A_{1}^{-1}$ и $A_{2}^{-1}$. Если адамарово произведение $A=A_{1} \circ A_{2}$ невырожденно, то матрица $A^{-1}$ также верхняя хессенбергова.

Действительно, если условия (24) выполняются для $A_{1}$ и $A_{2}$, то они верны и для $A$.

Рассмотрим теперь адамарово произведение брауновской матрицы (3) и отраженной брауновской матрищы

$$
\left(\begin{array}{ccccc}
k_{1} & l_{2} & l_{3} & \ldots & l_{n} \\
k_{2} & k_{2} & l_{3} & \ldots & l_{n} \\
k_{3} & k_{3} & k_{3} & \ldots & l_{n} \\
\vdots & \vdots & \vdots & \ddots & \vdots \\
k_{n} & k_{n} & k_{n} & \ldots & m_{n}
\end{array}\right)
$$

Полученная матрица имеет более общую форму

$$
\left(\begin{array}{ccccc}
k_{1} a_{1} & l_{2} b_{1} & l_{3} b_{1} & \ldots & l_{n} b_{1} \\
k_{2} a_{1} & k_{2} a_{2} & l_{3} b_{2} & \ldots & l_{n} b_{2} \\
k_{3} a_{1} & k_{3} a_{2} & k_{3} a_{3} & \ldots & l_{n} b_{3} \\
\vdots & \vdots & \vdots & \ddots & \vdots \\
k_{n} a_{1} & k_{n} a_{2} & k_{n} a_{3} & \ldots & m_{n} a_{n}
\end{array}\right)
$$

по сравнению с матрищей в [4], поскольку ни один сомножитель адамарова произведения не обязан быть симметричным. Условие неразложимости (23) для матрицы (27) принимает вид

$$
k_{n} a_{1} \neq 0
$$

Это условие обеспечивает ранг, не меньший единицы, всем подматрицам, участвующим в проверке соотношений (22). С другой стороны, указанные подматрищы отличаются от аналогичных подматриц для матрицы (3) умножением строк на соответствующие числа $k_{i}$. Таким образом, эти подматрицы имеют ранг 1 , что приводит нас к следующему утверждению.

Теорема 6. Если адамарово произведение А брауновской матрицы, удовлетворяюшей условиям (24), и отражсеной брауновской матрицы типа (26) невырожденно, то оно имеет в качестве $A^{-1}$ верхнюю матрицу Хессенберга. Эта матрича неразложима, если выполнено условие (28).

Теорема 6 объясняет результат из [4], процитированный во введении статьи, заодно обобщая его путем отказа от симметрии сомножителей адамарова произведения. Рассмотрим, например, произведение матрищы (25) и матрищы

$$
\left(\begin{array}{lllll}
5 & 1 & 5 & 2 & 3 \\
4 & 4 & 5 & 2 & 3 \\
3 & 3 & 3 & 2 & 3 \\
2 & 2 & 2 & 2 & 3 \\
1 & 1 & 1 & 1 & 3
\end{array}\right)
$$


Помимо несимметричности, отметим в этой матрице неравенство элемента $(5,5)$ внедиагональным элементам пятой строки. Это аналог отказа от условия $a_{1}=c_{1}$ для стандартных брауновских матриц. Матрица, обратная к адамарову произведению, имеет хессенбергову форму

$$
\left(\begin{array}{ccccc}
\frac{4}{137} & -\frac{42}{1507} & -\frac{351}{1507} & \frac{162}{685} & \frac{216}{685} \\
-\frac{1}{137} & -\frac{195}{1507} & -\frac{3029}{1507} & \frac{1398}{685} & \frac{1864}{685} \\
0 & \frac{1}{11} & \frac{6}{11} & -\frac{3}{5} & -\frac{4}{5} \\
0 & 0 & 1 & -1 & -1 \\
0 & 0 & 0 & \frac{1}{15} & -\frac{2}{15}
\end{array}\right)
$$

4. Вычисление матрицы, обратной к брауновской. Между брауновскими и трехдиагональными матрищами существует тесная взаимосвязь, описываемая приводимой ниже теоремой 7 . Пусть $P$ - нижнетреугольная $n \times n$ матрица с элементами

$$
p_{i j}= \begin{cases}1, & i=j, \\ -1, & j=i-1, \\ 0, & \text { для прочих } i, j .\end{cases}
$$

Теорема 7 ([13], [14]). Матрица $A$ порядка $n$ тогда и только тогда является брауновской, когда матрича

$$
C=P A P^{T}
$$

трехдиагональная.

Переход от матрищы $A$ к матрище $C$ требует, как мы сейчас увидим, всего лишь $O(n)$ аддитивных операций. Поэтому формула (29) указьвает один из возможных $O(n)$-алгоритмов для решения линейных систем с невырожденньми брауновскими матрищами.

Пусть

$$
C=\left(\begin{array}{cccccc}
p_{1} & r_{1} & 0 & \ldots & \ldots & 0 \\
s_{1} & p_{2} & r_{2} & 0 & & 0 \\
0 & s_{2} & p_{3} & r_{3} & \ddots & \vdots \\
\vdots & \ddots & \ddots & \ddots & \ddots & 0 \\
0 & & 0 & & & r_{n-1} \\
0 & \ldots & \ldots & 0 & s_{n-1} & p_{n}
\end{array}\right)
$$

есть матрица, полученная из матрицы (1) по формуле (29). Установим связь между параметрами $p_{i}, r_{i}, s_{i}$ и $a_{i}, b_{i}, c_{i}$ обеих матриц.

Левое умножение $A$ на матрицу $P$ дает верхнюю хессенбергову матрицу

$$
E=\left(\begin{array}{cccccc}
a_{1} & b_{1} & \ldots & b_{1} & b_{1} & b_{1} \\
c_{1}-a_{1} & a_{2}-b_{1} & \ldots & b_{2}-b_{1} & b_{2}-b_{1} & b_{2}-b_{1} \\
\ldots \ldots & \ldots & \ldots & \ldots \ldots \ldots \ldots \ldots \ldots \ldots \ldots \ldots \ldots \ldots \ldots \ldots \ldots \ldots \ldots \ldots \\
0 & 0 & \ldots & a_{n-2}-b_{n-3} & b_{n-2}-b_{n-3} & b_{n-2}-b_{n-3} \\
0 & 0 & \ldots & c_{n-2}-a_{n-2} & a_{n-1}-b_{n-2} & b_{n-1}-b_{n-2} \\
0 & 0 & \ldots & 0 & c_{n-1}-a_{n-1} & a_{n}-b_{n-1}
\end{array}\right)
$$


Обратим внимание на то, что $E$ сохранила брауновскую структуру в своей верхнетреугольной части. Другое примечательное обстоятельство состоит в том, что для матрицы $A$, удовлетворяющей условиям Валви

$$
c_{i}=a_{i}, \quad i=1, \ldots, n-1
$$

матрица $E$ является треугольной. В этом случае $E$ (а стало быть, и $A$ ) невырожденна тогда и только тогда, когда

$$
a_{1} \neq 0, \quad a_{i} \neq b_{i-1}, \quad i=2, \ldots, n
$$

Правое умножение $E$ на матрицу $P^{T}$ не затронет поддиагональных элементов. В итоге мы приходим к формулам

$$
\begin{gathered}
p_{1}=a_{1}, \\
p_{i}=\left(a_{i}+a_{i-1}\right)-\left(b_{i-1}+c_{i-1}\right), \quad i=2, \ldots, n, \\
r_{i}=b_{i}-a_{i}, \quad i=1, \ldots, n-1, \\
s_{i}=c_{i}-a_{i}, \quad i=1, \ldots, n-1 .
\end{gathered}
$$

Если $A$ невырожденна, то из формулы (29) вьводим

$$
A^{-1}=P^{T} C^{-1} P
$$

Используя это соотношение и результаты раздела 2, установим общий вид матрищы $B$, обратной к брауновской матрице $A$. Для простоты, наложим на $A$ ограничение, заключающееся в том, что отвечающая ей матрица (30) должна быть неразложимой. Согласно (35), (36) это ограничение можно задать неравенствами

$$
b_{i} \neq a_{i}, \quad c_{i} \neq a_{i}, \quad i=1, \ldots, n-1 .
$$

Как следует из раздела 2 , матрица $C^{-1}$ описывается с помощю четырех $n$-мерных векторов $x, y, u, v$ таким образом, что

$$
\left(C^{-1}\right)_{i j}= \begin{cases}u_{i} v_{j}, & j \geqslant i \\ y_{i} x_{j}, & i \geqslant j .\end{cases}
$$

При этом

$$
u_{i} v_{i}=x_{i} y_{i}, \quad i=1,2, \ldots, n \text {. }
$$

Вычисление вектора $u$ предполагает (см. (16)) решение ленточной треугольной системы линейных уравнений

$$
C_{n-1} \hat{u}=b,
$$


где

$$
C_{n-1}=\left(\begin{array}{cccccc}
r_{1} & 0 & \ldots & \ldots & \ldots & 0 \\
p_{2} & r_{2} & 0 & & & \vdots \\
s_{2} & p_{3} & r_{3} & \ddots & & \vdots \\
\vdots & \ddots & \ddots & \ddots & 0 & \vdots \\
\vdots & & & p_{n-2} & r_{n-2} & 0 \\
0 & \ldots & \ldots & s_{n-2} & p_{n-1} & r_{n-1}
\end{array}\right)
$$

a

$$
b^{T}=\left(p_{1} s_{1} 0 \ldots 0\right)
$$

Нетривиальные компоненты вектора $v$ определяются путем решения верхнетреугольной системы линейных уравнений

$$
C_{n-1}^{T} \hat{v}=c,
$$

где

$$
c^{T}=\left(0 \ldots 0 s_{n-1} p_{n}\right)
$$

Число $s$, входящее в определение вектора $u$, находится как

$$
s=-c^{T} \hat{u}
$$

или как

$$
s=-v^{T} b
$$

Аналогичным образом вычисляются векторы $x$ и $y$. Роль матрицы $C_{n-1}$ при этом выполняет матрица

$$
\left(\begin{array}{cccccc}
s_{1} & 0 & \ldots & \ldots & \ldots & 0 \\
p_{2} & s_{2} & 0 & & & \vdots \\
r_{2} & p_{3} & s_{3} & \ddots & & \vdots \\
\vdots & \ddots & \ddots & \ddots & 0 & \vdots \\
\vdots & & & p_{n-2} & s_{n-2} & 0 \\
0 & \ldots & \ldots & r_{n-2} & p_{n-1} & s_{n-1}
\end{array}\right)
$$

с соответствующими изменениями в векторах $b$ и $c$.

Решение систем (41) и (44) обходится, как легко видеть, в $O(n)$ арифметических операций. Поэтому, если не требуется формирования матрицы $C^{-1}$ в явном виде, для вычисления определяющих ее векторов $x, y, u, v$ достаточно $O(n)$ операций. Нам остается показать, что и матрицу $A^{-1}$ можно записать с помощью компонент этих векторов.

Выполняя преобразования, предусматриваемые формулой (37), нетрудно проверить, что элементы матрищы $B=A^{-1}$ выражаются формулами

$$
b_{i j}= \begin{cases}\left(u_{i+1}-u_{i}\right) v_{i+1}-\left(y_{i+1}-y_{i}\right) x_{i}, & i=j=1, \ldots, n-1, \\ u_{n} v_{n}=x_{n} y_{n}, & i=j=n \\ \left(u_{i+1}-u_{i}\right)\left(v_{j+1}-v_{j}\right), & j>i, \\ \left(x_{j+1}-x_{j}\right)\left(y_{i+1}-y_{i}\right), & i>j .\end{cases}
$$


Для двух последних формул мы принимаем определение

$$
v_{n+1}=y_{n+1}=0 \text {. }
$$

Мы видим, что любой диагональный элемент матрицы $B$ может быть восстановлен из компонент векторов $x, y, u, v$ посредством не более чем пяти арифметических операций. Для восстановления внедиагонального элемента $b_{i j}$ достаточно трех операций. Наконец, если ввести векторы $\tilde{x}, \tilde{y}, \tilde{u}, \tilde{v}$ с помошью соотношений

$$
\begin{aligned}
& \hat{x}_{i}=\left\{\begin{array}{ll}
x_{i+1}-x_{i}, & i \neq n, \\
x_{i}, & i=n,
\end{array} \quad \hat{y}_{i}=y_{i+1}-y_{i}, \quad i=1, \ldots, n,\right. \\
& \hat{u}_{i}=\left\{\begin{array}{ll}
u_{i+1}-u_{i}, & i \neq n, \\
u_{i}, & i=n,
\end{array} \hat{v}_{i}=v_{i+1}-v_{i}, \quad i=1, \ldots, n,\right.
\end{aligned}
$$

то внедиагональные элементы матрицы $B$ могут быть записаны формулами

$$
b_{i j}=\left\{\begin{array}{l}
\hat{u}_{i} \hat{v}_{j}, \quad j>i, \\
\hat{y}_{i} \hat{x}_{j}, i>j,
\end{array}\right.
$$

подчеркивающими родство с обращением трехдиагональной матрицы (см. $(21))$.

\section{СПИСОК ЦИТИРОВАННОЙ ЛИТЕРАТУРЫ}

[1] Gregory R. T., Karney D. L. A collection of matrices for testing computational algorithms. John Wiley, 1969.

[2] Valvi F. Explicit presentation of the inverses of some types of matrices // J. Inst. Maths. Applics. 1977. V. 19. P. 107-117.

[3] Valvi F. N., Geroyannis V.S. Analytic inverses and determinants for a class of matrices // IMA J. Numer. Analysis. 1987. V. 7. P. 123-128.

[4] Valvi F. N. The Hadamard product of two Brownian matrices: analytic inverse and determinant // J. Austral. Math. Soc. Ser. B. 1995. V. 36. P. 493-497.

[5] Ėidelman Yu.S., Gohberg I. On a new class of structured matrices // Integral Equations Operator Theory. 1999. V. 34. № 3. P. 293-324.

[6] Tyrtyshnikov E. E. // Mosaic ranks and skeletons matrices. Numerical analysis and its applications. (Rousse, 1996). Lecture Notes in Computer Science. 1997. V. 1196. P. 505-516.

[7] Fiedler M., Markham T. L. Rank-preserving diagonal completions of a matrix // Linear Algebra Appl. 1987. V. 85. P. 49-56.

[8] Fiedler M. Structure ranks of matrices // Linear Algebra Appl. 1993. V. 179. P. 119-127.

[9] Хорн Р., Джонсон Ч. Матричньй анализ. М.: Мир, 1990.

[10] Elsner L. Some observations on inverses of band matrices and low-rank perturbations of triangular matrices // Acta Technica Acad. Sci. Hung. 1997-99. V. 108. № 1-2. P. 41-48.

[11] McDonald J. J., Nabben R., Neumann M., Schneider H., Tsatsomeros M. Inverse tridiagonal Z-matrices // Linear and Multilinear Algebra. 1998. V. 45. P. 75-97.

[12] Asplund E. Inverses of matrices $\left\{a_{i j}\right\}$ which satisfy $a_{i j}=0$ for $j>i+p / /$ Mathematica Scandinavica. 1959. V. 7. P. 57-60.

[13] Gover M. J. C., Barnett S. Brownian matrices: properties and extensions // Int. J. Syst. Sci. 1986. V. 17. № 2. P. 381-386.

[14] Krishna H., Morgera S. D. Fast $O(n)$ complexity algorithms for diagonal innovation matrices // IEEE Trans. Acoust., Speech, Signal Process. 1984. V. 32. P. 1189-1193. 